\title{
Correction to: Mechanisms of multiple neurotransmitters in the effects of Lycopene on brain injury induced by Hyperlipidemia
}

\author{
Weichun Yang, Ziyi Shen, Sixian Wen, Wei Wang and Minyu Hu*
}

\section{Correction}

Following publication of the original article [1] it came to the attention of the Research Integrity Group that the following corrections were required:

- In the "Consent for publication" section the sentence "All co-authors and participants have given their consent for publication of this article in Lipids in Health and Disease" should be replaced with "Not applicable"

- The section titled "Competing of interests" should be corrected to "Competing interests"

- The title of the section labeled "Author' contributions" should be changed to "Authors' contributions"

- The sentence "None of the authors reported a conflict of interest related to this study" should be removed from the end of the "Authors' contributions" section.

The original article has been corrected.

Received: 12 March 2018 Accepted: 12 March 2018

Published online: 03 April 2018

\section{Reference}

1. Yang W, Shen Z, Wen S, Wang W, Minyu H. Mechanisms of multiple neurotransmitters in the effects of Lycopene on brain injury induced by Hyperlipidemia. Lipids Health Dis. 2018;17:13. https:/doi.org/10.1186/ s12944-018-0660-5.

\footnotetext{
* Correspondence: huminyu@csu.edu.cn

Department of Nutrition and Food Hygiene, Xiangya School of Public Health, Central South University, 110 Xiangya Road, Changsha 410078, China
} 